\title{
CORRECTIONS
}

\section{Associations of fat and carbohydrate intake with cardiovascular disease and mortality: prospective cohort study of UK Biobank participants}

In this article by Ho and colleagues (BMJ 2020;368, doi:10. 1136/bmj.m688, published 18 March 2020) the data for two of the body mass index categories in table 1 were misaligned. The data in the rows for underweight and normal weight should be transposed. 